\title{
Knowledge and Practices of Mothers: Infant and Young Child's Feeding in Chowk Azam, the Punjab, Pakistan
}

\author{
Anaam Arif ${ }^{1, ~}$, Ejaz Ahmad Khan", Abid Hussain ${ }^{2}$, Muhammad Awais Arif ${ }^{3}$ \\ ${ }^{1}$ Department of Public Health, Health Services Academy, Islamabad, Pakistan \\ ${ }^{2}$ Independent Consultant, Islamabad, Pakistan \\ ${ }^{3}$ Department of Medical Sciences, University of Medical Sciences, Cienfuegos, Cuba
}

Email address:

anamarif72@gmail.com (A. Arif)

\section{To cite this article:}

Anaam Arif, Ejaz Ahmad Khan, Abid Hussain, Muhammad Awais Arif. Knowledge and Practices of Mothers: Infant and Young Child's Feeding in Chowk Azam, the Punjab, Pakistan. Journal of Food and Nutrition Sciences. Vol. 3, No. 6, 2015, pp. $236-239$.

doi: $10.11648 /$ j.jfns.20150306.16

\begin{abstract}
In order to investigate the knowledge and practices of mothers regarding breast feeding and complementary feeding and to assess their compliance with the health guidelines, we conducted a cross-sectional survey of infant complementary feeding and breast feeding practices was conducted in a sample of 230 mothers. Data was collected systematically from rural areas of Chowk Azam. A validated questionnaire comprising of questions pertaining to sociodemographic profile, knowledge and practices of breast feeding and complementary feeding practices was used. The data was analyzed using SPSS version 20 and chi square test was applied.Our study resultsrevealed thatonly $18 \%$ mothers were practicing complementary feeding at the recommended age. About $72 \%$ of the respondents started complementary feeding before the recommended age of six months and 3\% mothers started delayed complementary feeding. Around $6.5 \%$ mothers had children less than six months of age and did not start complementary feeding yet. Almost half of the respondents $56.3 \%$ were using the homemade complementary food, while less than a fourth i.e. $19.5 \%$ mothers were giving commercially prepared food to their children and rest of one-fourth mothers $24.2 \%$ were giving both types of food. We conclude that knowledge and practices of mothers pertaining to breast feeding and complementary feeding practices are at mixed levels, and their understanding aboutexact time of starting complementary feeding is suboptimal. There is a need to create awareness among mothers regarding theappropriate time of starting complementary feeding so that health status of the infants and children can be ameliorated.
\end{abstract}

Keywords: Complementary Feeding, Pakistan, Knowledge, Breast Feeding

\section{Introduction}

Feeding practices of infants are chief determinants of future physical and mental well- being because of rapid growth and development of tissues during the first year of life [1].Health status of child is immensely affected by the breastfeeding and complementary feeding practices. Childhood under nutrition is quite common in low and middle income countries and it is one of the contributing factors for child mortality. It has been estimated that around the globe about 2.1 million deaths in children less than 5 years of age occur just because of wasting, stunting and intrauterine growth restriction [2]. Child health is negatively influenced by both under nutrition and inappropriate stimulation and as a result imposes long term impacts by adversely affecting the educational and economical outcomes of child [3, 4]. After six months of age exclusive breast feeding is insufficient to fulfill the nutritional requirements of the rapidly growing child as a result incidence of stunting are highest in this age [5].

Therefore there is need to introduce the solid food at the age of six months $[6,7]$.Complementary food should be safe and nutritious and it should be introduced at appropriate time and in adequate amount [8]. In Pakistan complementary feeding practices are not according to the health guidelines and suboptimal feeding practices have been observed in various parts of the country $[9,10]$. The aim of present study is to describe the breast feeding and complementary feeding practices among mothers at Chowk Azam, Pakistan and to assess their compatibility with health guidelines. 


\section{Materials and Methods}

\subsection{Study Design}

Cross sectional study was conducted that was descriptive and exploratory in nature. Breast feeding and complementary feeding practices among motherswere determined and their compatibility with health guidelines was assessed. The risk factors for early and delayed initiation of complementary feeding practices were also identified.

\subsection{Subjects}

The sample was collected from rural community of Chowk Azam and systematic sampling technique was used. Mothers having child up to 2 years of age or below were included in the study. About 230mothers participated in the study.

\subsection{Data Collection Tool}

A validated closeended, structured questionnaire was used to collect the data. Questionnaire was first piloted with 10 participants among community. The questionnaire comprised of sociodemographic characteristics of the study participants including education level of mother, family income and age. Study variables comprised of time of starting complementary feeding, frequency of complementary feed and type of complementary food. Questionnaire was translated into local language so that study participants could understand and respond accurately. Informed consent form was obtained prior to interviews.

\subsection{Data Analysis}

Data analysis was carried out by using SPSS version 20 and chi square test was applied to see the association between dependent and independent variables.Descriptive summary statistics such as frequencies and percentages were computed for statically important variables. The inferential analysis at p-value $<0.05$ was carried out using Chi-Square test and study variables were cross tabulated to assess the level of association of categorical/nominal variables. Results were presented in Graphic and tabular forms.

\section{Results}

\subsection{Demographic Data}

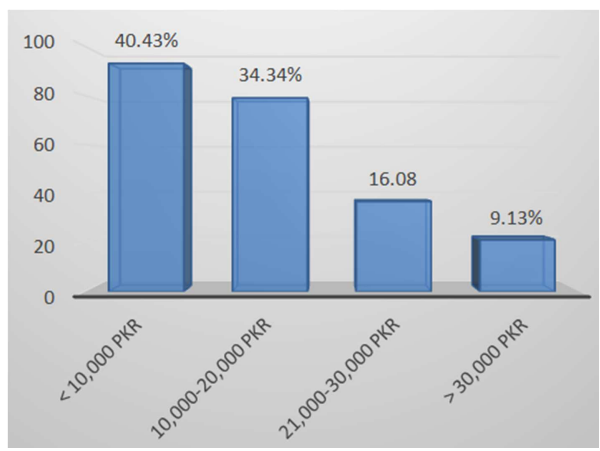

Figure 1. Monthly income of the family.
Out of 230 mothers who participated in the study about $37 \%$ mothers were illiterate and around $33.5 \%$ of the participants were having primary level education, $16.5 \%$ obtained middle level education, whereas $10 \%$ had high school education. Only 3\% mothers received graduation degree.

\subsection{Breast Feeding Practices}

In a response to a question about duration of exclusive breastfeeding only $11.7 \%$ mothers correctly answered that it is after six months. More than two-third $71.3 \%$ of the respondents said that exclusive breastfeeding is recommended only for four months.

Table 1. Sociodemographic characteristics of the respondents.

\begin{tabular}{lll}
\hline Variable & Frequency $(\mathbf{n}=\mathbf{2 3 0})$ & Percentage $(\%)$ \\
\hline Education level of mother & & \\
Illiterate & 85 & 37 \\
Primary & 77 & 33.5 \\
Middle & 38 & 16.5 \\
High & 23 & 10 \\
HSSC & 4 & 1.7 \\
Graduation & 2 & 0.86 \\
Religious & 1 & 0.4 \\
Monthly income of family & & \\
$<10,000$ PKR & 93 & 40.4 \\
10,000-20,000 & 79 & 34.3 \\
$21,000-30,000$ & 37 & 16.1 \\
$>30,000$ & 21 & 9.1 \\
\hline
\end{tabular}

\subsection{Complementary Feeding Practices}

Table 2. Complementary feeding practices among the respondents.

\begin{tabular}{lc}
\hline Variable & Frequency (percentage)(n=230) \\
\hline Started complementary feeding & $215(93.4)$ \\
Yes & $15(6.5)$ \\
No & $167(77.7)$ \\
Age of children whent started complementary feeding $(\mathrm{n}=215)$ \\
$<6$ months & $41(19)$ \\
At 6 months & $7(3.3)$ \\
$>6$ months & $138(82.6)$ \\
Reasons of early complementary feeding $(\mathrm{n}=167)$ \\
Didn't know when to start CF & $23(13.8)$ \\
Breast milk was insufficient & $6(3.6)$ \\
Sickness of mother & $2(28.6)$ \\
Reasons for delayed complementary feeding $(\mathrm{n}=7)$ \\
Didn't know exactly when to start & $3(42.9)$ \\
Feeling that breast-milk is sufficient & $1(14.3)$ \\
Family elders advised to start late & $1(14.3)$ \\
Feeling that child was not able to & $29(13.5)$ \\
digest & $63(29.3)$ \\
Food source being given to children $(\mathrm{n}-215)$ \\
Commercially prepared & $42(19.5)$ \\
Homemade & $121(56.3)$ \\
Both & $52(24.2)$ \\
No of complementary feeding per day $(\mathrm{n}-215)$ \\
One meal/day & \\
2-3 meals/day & \\
$>3$ meals/day & \\
\hline &
\end{tabular}


Only about $18 \%$ of mothers had started complementary feeding at recommended age of the children. $72.6 \%$ of the respondents had started complementary feeding before the recommended age of six months. 3\% had started delayed complementary feeding i.e. at more than six months of age. $6.5 \%$ of the respondents had not started complementary feeding to their children (i.e. the age of their children was less than 6 months). Around 56.3\% mothers were giving homemade food to their children, $19.5 \%$ were giving commercially prepared food and $24.2 \%$ were giving both types of food. Most of $57.2 \%$ of the mothers were giving more than 3 meals per day and $13.5 \%$ were giving one meal per day.

\section{Discussion}

The study results revealed that in Pakistan, contrary to health guidelines, the practice of feeding children with solid or semi solid food starts quite early in life [11]. A large percentage of children i.e. about $72.6 \%$ children were receiving complementary feed below age of 6 months. Only $18 \%$ children were given solid food at the recommended. However there was a minor fraction of children $3 \%$ who received solid food at greater than 6 months of age.

Lack of knowledge about exact time of starting complementary feeding was the main reason for suboptimal feeding practices [12].About $82.6 \%$ of those respondents who were practicing early complementary feeding didn't know when to start complementary feeding. Majority of these mothers were illiterate and they were having little knowledge about importance of complementary food and its impact on the child's health. . Other reasons included were mother's perception that breast milk is insufficient for children about $3.6 \%$ mothers said their sickness led them to start early complementary feeding. Approximately 3\% of mothers who started delayed feeding tried to justify that breast milk was enough for their babies. Other reasons stated by mothers for delayed complementary feeding were lack of knowledge about time of initiation of weaning (28.6\%), instructions from family members $(14.3 \%)$ and feeling of mothers that their children were not able to digest food other than breast-milk. Tabish et al also showed similar results; prevalence of suboptimal feeding practices is quite high among Pakistani mothers and there is need to develop interventions to control these alarming rates [13]. Asimilar study conducted by Shirely et al revealed that education level of mothers'effect time of introduction of complementary food. Shirley et al stated that educated mothers had more knowledge about significance of complementary food and they had better weaning practices compared to uneducated mothers. Another study conducted by Ali et al revealed there was significant improvement in complementary feeding practices among mothers after providing them health education.

Infant mortality rate and child mortality rate associated with the use of inappropriate complementary feeding practices are already high in Pakistan and there is need to resolve this issue on priority basis. Otherwise situation may get worse thus making it impossible for Pakistan to achieve it MDG 4 and 5 .

\section{Conclusion}

The high level of noncompliance with the health guidelines could conclude that lack of knowledge about complementary feeding is one of the main reasons for poor feeding practices. There is need to improve the health knowledge of mothers regarding the duration of exclusive breast feeding and time of initiation of complementary feeding. Health professional should be trained to properly counsel the mothers about frequency and adequacy of complementary food.

\section{References}

[1] Psasir.upm.edu.my. Infant Feeding Practices, Health And Nutritional Status: A Prospective Study Of Infants Seen At The University Of Malaya Medical Centre, Kuala Lumpur Universiti Putra Malaysia Institutional Repository [Internet]. 2015 [cited 19 June 2015]. Available from: http://psasir.upm.edu.my/11245/

[2] Imdad A, Yakoob M, Bhutta Z. Impact of maternal education about complementary feeding and provision of complementary foods on child growth in developing countries. BMC Public Health. 2011; 11(Suppl 3): S25.

[3] Christian P, Mullany L, Hurley K, Katz J, Black R. Nutrition and maternal, neonatal, and child health. Seminars in Perinatology. 2015; 39(5): 361-372.

[4] Arabi M, Frongillo E, Avula R, Mangasaryan N. Infant and Young Child Feeding in Developing Countries. Child Development. 2012; 83(1): 32-45.

[5] Menon P. The crisis of poor complementary feeding in South Asia: where next? Maternal \& Child Nutrition. 2011; 8: 1-4.

[6] Fewtrell M, Wilson D, Booth I, Lucas A. Six months of exclusive breast feeding: how good is the evidence? BMJ. 2010; 342(jan13 1): c5955-c5955.

[7] Hanif $\mathrm{H}$. Trends in breastfeeding and complementary feeding practices in Pakistan, 1990-2007. IntBreastfeed J. 2011; 6(1): 15 .

[8] Mennella J, Trabulsi J. Complementary Foods and Flavor Experiences: Setting the Foundation. Annals of Nutrition and Metabolism. 2012; 60(s2):40-50.

[9] Huh S, Rifas-Shiman S, Taveras E, Oken E, Gillman M. Timing of Solid Food Introduction and Risk of Obesity in Preschool-Aged Children. PEDIATRICS. 2011; 127(3): e544e551.

[10] Impact of Maternal Education about Complementary Feeding on Their Infants' Nutritional Outcomes in Low- and Middleincome Households: A Community-based Randomized Interventional Study in Karachi, Pakistan. Journal of Health, Population and Nutrition [Internet]. 2015; 32(4). Available from:

http:/www.ncbi.nlm.nih.gov/pmc/articles/PMC4438693/ 
[11] Zafar M, Fatmi Z, Shafi K. Determinants of child feeding practices in Pakistan; secondary data analysis of demographic and health survey 2006-07. J Med NutrNutraceut. 2014; 3(2): 198.

[12] Bhatia R, Jain U. Knowledge, attitude, practices and misconceptions among mothers regarding complementary feeding. Int J Med Sci Public Health. 2014; 3(11): 1.

[13] Hazir T, Akram D, Nisar Y, Kazmi N, Agho K, Abbasi S et al. Determinants of suboptimal breast-feeding practices in Pakistan. Public Health Nutr. 2012; 16(04): 659-672. 